15

\title{
Оценка производства ультрахолодных нейтронов в проекте источника на реакторе ВBP-М
}

\author{
(C) М.С. Онегин, А.П. Серебров, А.К. Фомин, В.А. Лямкин \\ ${ }^{1}$ Национальный исследовательский центр „Курчатовский институт“ \\ ${ }^{2}$ Петербургский институт ядерной фризики им. Б.П. Константинова, \\ 188300 Гатчина, Ленинградская область, Россия \\ I e-mail: serebrov@pnpi.spb.ru
}

(Поступило в Редакцию 14 июня 2016 г.)

\begin{abstract}
Представлены расчеты, связанные с проектированием источника ультрахолодных нейтронов со сверхтекучим гелием на реакторе ВВР-М. Проведены расчеты скорости генерации ультрахолодных нейтронов при различном наполнении камеры предзамедлителя, а также определена зависимость этой скорости от температуры гелия. При заполнении камеры предзамедлителя жидким дейтерием в ортосостоянии скорость генерации ультрахолодных нейтронов практически постоянна в диапазоне изменения температуры гелия от 1.0 до $1.5 \mathrm{~K}$ и достигает $3.1 \cdot 10^{3} \mathrm{~cm}^{-3} \mathrm{~s}^{-1}$.
\end{abstract}

DOI: 10.21883/JTF.2017.04.44324.1924

\section{Введение}

Источник ультрахолодных нейтронов (УХН) на реакторе ВВР-М [1] в настоящее время находится в фазе проектирования. Он будет смонтирован в тепловой колонне реактора внутри графитового блока и будет состоять из камеры предзамедлителя, заполняемой жидким дейтерием, и самой гелиевой камеры, заполненной сверхтекучим гелием. Температура в гелиевой камере определяется уровнем тепловыделения в ней за счет радиационного излучения и теплопритоков, а также возможностями теплосъема. Оценки и натурные эксперименты показывают, что ожидаемая температура гелия в камере будет лежать в диапазоне от 1.2 до $1.4 \mathrm{~K}$. Температура жидкого дейтерия в камере предзамедлителя будет поддерживаться на уровне $20 \mathrm{~K}$. На этапе ввода в эксплуатацию камера предзамедлителя будет первоначально заполняться холодным гелием или другими газами. И только на конечном этапе она будет заполнена жидким дейтерием. Целями настоящей работы являлись расчеты скорости накопления УХН при различном наполнении камеры предзамедлителя, а также определение зависимости этой скорости от температуры гелия.

\section{1. Жидкий гелий как производитель YXH}

В жидком гелии, согласно теории Ландау, возможны однофононные возбуждения. Дисперсионная кривая зависимости энергии этих возбуждений от переданного импульса представлена на рис. 1. На этом же рисунке приведена зависимость энергии нейтрона от его импульса. При импульсе $\sim 0.7 \AA^{-1}$ эти кривые пересекаются, т. е. нейтрон может передать всю свою энергии фонону. При этом нейтрон становится ультрахолодным.
Скорость рождения УХН вычисляется по формуле [2]:

$$
\begin{aligned}
P_{m p} & =\int_{0}^{R_{c}} P\left(E_{u}\right) d E_{u} \\
& =N_{\mathrm{He}} \sigma_{b} \alpha^{2}\left[\int \frac{d \Phi\left(k_{1}\right)}{d E} S\left(k_{1}, \omega=\frac{\alpha k_{1}^{2}}{2}\right) d k_{1}\right] \frac{k_{c}^{3}}{3} .
\end{aligned}
$$

Здесь $S$ - структурный фактор (известен экспериментально), $\sigma_{b}=1.1 b-$ связанное сечение рассеяния на гелии, $N_{\mathrm{He}}$ - число ядер гелия в $\mathrm{cm}^{3}, E_{c} k_{c}-$ критическая энергия и волновой вектор УХН для материала стенок камеры, $\alpha=\hbar / m=4.14 \mathrm{MeV} / \AA^{2}, k_{1}$ - волновой вектор нейтрона. График зависимости структурного фактора для однофононных (первый пик на графике) и многофононных (размытый пик в области импульсов 0.9-1.9 $\AA^{-1}$ ) возбуждений в свертекучем жидком гелии представлен на рис. 2. Таким образом, как следует из

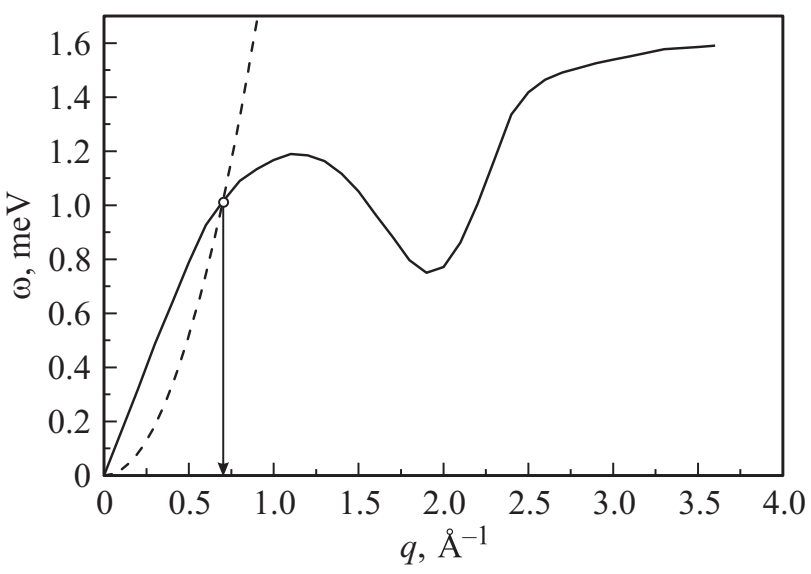

Рис. 1. Дисперсионная кривая фононных возбуждений в жидком сверхтекучем гелии (сплошная кривая), зависимость энергии нейтрона от импульса (штриховая кривая). 


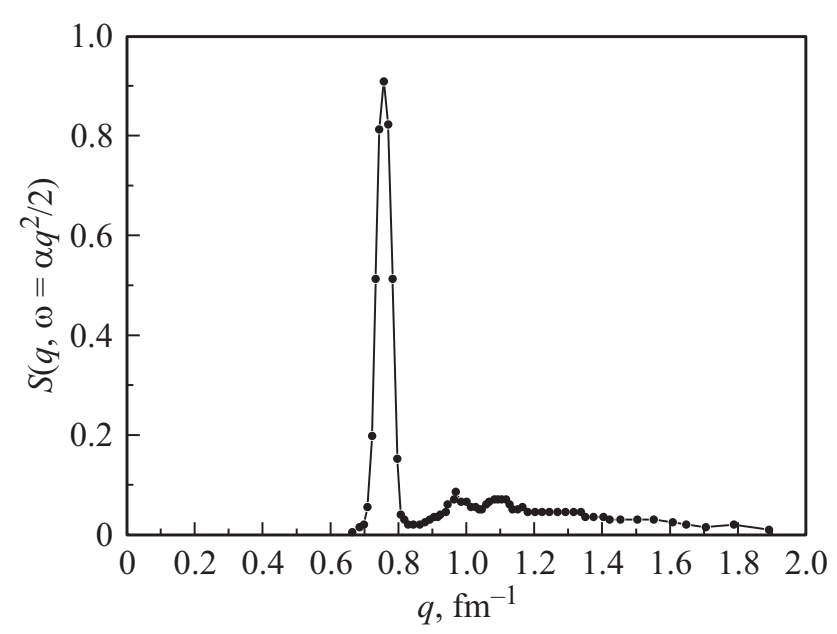

Рис. 2. Структурный фактор фононных возбуждений в сверхтекучем гелии.

формулы (1), для производства УХН в сверхтекучем жидком гелии наиболее ценны нейтроны с длиной волны $9 \AA$, так что переданный импульс при их остановке равен $0.7 \AA^{-1}$. Для максимальной производительности УХН источник должен быть оптимизирован на достижение максимальной плотности потока нейтронов с такой длиной волны в гелиевой камере.

Интегрирование в формуле (1) включает не только максимум структурной функции в районе $0.7 \AA^{-1}$, но и большие переданные импульсы, где структурная функция отлична от нуля. Полный интеграл определяет скорость генерации УХН с учетом многофононности $p_{m p}$, тогда как интеграл только по области переданных импульсов в районе пика дает скорость генерации УХН в однофононном приближении $-P_{1 p}$.

В формуле (1) можно перейти от интегрирования по переданному импульсу к интегрированию по длине волны нейтрона:

$$
P_{m p}=N_{\mathrm{He}} \sigma_{b} \frac{\alpha k_{c}^{3}}{6 \pi} \int_{0}^{\infty} \Phi(\lambda) \lambda d \lambda,
$$

где

$$
s(\lambda)=\hbar \int S(q, \omega) \delta\left(\hbar \omega-E_{n}\right) d \omega,
$$

а $\Phi(\lambda)$ - плотность потока нейтронов с длиной волны $\lambda$. Энергия нейтрона связана с длиной волны формулой $E_{n}=h^{2} /\left(2 m \lambda^{2}\right)$.

\section{2. Предзамедлитель}

Гелий эффективно конвертирует нейтроны с длиной волны $9 \AA$ в УХН. Таким образом, для эффективной работы гелиевой камеры она должна находиться в интенсивном потоке нейтронов с длиной волны $9 \AA$. Поэтому в полость теплого графита тепловой колонны необходимо поместить дополнительную камеру при низкой температуре с тем, чтобы эффективно понизить температуру нейтронов в гелиевой камере. В качестве рабочего тела предзамедлителя был выбран жидкий дейтерий при температуре около $20 \mathrm{~K}$, стандартно использующийся в источниках холодных нейтронов и УХН.

Схема источника УХН в тепловой колонне представлена на рис. 3. Камера предзамедлителя окружает гелиевую камеру внутри вакуумного контейнера. Запуск источника УХН в эксплуатацию будет производиться по этапам, так что на первом этапе будет заполняться только гелиевая камера. В последующем на этапах тестирования дейтериевая камера может быть заполнена газом при различных температурах. В качестве таких газов можно использовать водород или дейтерий в различном состоянии (орто- или пара-). В данном разделе рассчитывается скорость генерации УХН в гелиевой камере при различном заполнении дейтериевой камеры.

\section{1. Генерация УХН в источнике без предзамедлителя}

Спектр нейтронов в гелиевой камере при незаполненной дейтериевой и гелиевой камерах представлен на рис. 4 пунктиром. Заполнение гелиевой камеры жидким гелием увеличивает долю холодных нейтронов. Жидкий гелий рассматривался для двух температур 1.0 и $1.5 \mathrm{~K}$. Транспорт нейтронов в гелиевой камере рассчитывался с учетом рассеяния на атомах гелия в жидкости, используя нейтронные библиотеки для программы MCNP, подготовленные для рассматриваемой температуры при помощи кода NJOY [3]. При генерации библиотеки использовался экспериментальный структурный фактор для гелия, измеренный при температурах 1.0 и $1.5 \mathrm{~K}$ [4].

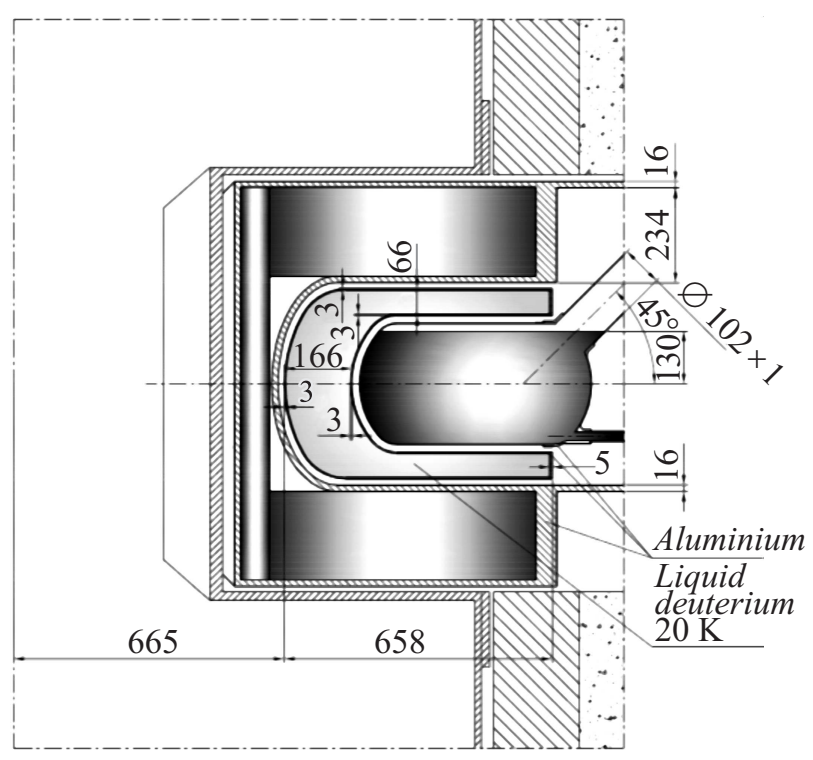

Рис. 3. Схема расположения элементов источника УХН в тепловой колонне. 
Скорость генерации УХН при разном заполнении камеры предзамедлителя

\begin{tabular}{|c|c|c|c|c|c|}
\hline $\begin{array}{c}\text { Заполнение } \\
\text { дейтериевой камеры }\end{array}$ & $\begin{array}{c}\text { Температура } \\
\text { жидкого гелия, K }\end{array}$ & $\begin{array}{c}\text { Плотность потока } 9 \AA \\
\text { нейтронов, } \mathrm{cm}^{-2} \mathrm{~s}^{-1} \AA^{-1}\end{array}$ & $\begin{array}{c}P_{m p} \\
\mathrm{~cm}^{-3} \mathrm{~s}^{-1}\end{array}$ & $\begin{array}{c}P_{1 p} \\
\mathrm{~cm}^{-3} \mathrm{~s}^{-1}\end{array}$ & $P_{m p} / P_{1 p}$ \\
\hline- & 1.0 & $4.9 \cdot 10^{8}$ & 115 & 34 & 3.4 \\
\hline - & 1.5 & $3.8 \cdot 10^{8}$ & 101 & 24.5 & 4.1 \\
\hline $\mathrm{H}_{\text {ort }}$, газ & 1.0 & $4.0 \cdot 10^{9}$ & 565 & 255 & 2.2 \\
\hline $\mathrm{H}_{\text {ort, }}$ газ & 1.5 & $3.6 \cdot 10^{9}$ & 530 & 230 & 2.3 \\
\hline $\mathrm{H}_{\text {para }}$, газ & 1.0 & $2.4 \cdot 10^{9}$ & 410 & 150 & 2.7 \\
\hline $\mathrm{H}_{\text {para }}$, газ & 1.5 & $2.1 \cdot 10^{9}$ & 380 & 130 & 2.9 \\
\hline $\mathrm{D}_{\mathrm{ort}}$, газ & 1.5 & $5.4 \cdot 10^{8}$ & 132 & 34 & 3.8 \\
\hline $\mathrm{H}_{\text {para }}$, газ & 1.5 & $5.3 \cdot 10^{8}$ & 128 & 33 & 3.9 \\
\hline $\mathrm{Be}$ & 1.0 & $9.7 \cdot 10^{8}$ & 230 & 65 & 3.6 \\
\hline $\mathrm{Be}$ & 1.5 & $7.5 \cdot 10^{8}$ & 210 & 49 & 4.3 \\
\hline $\mathrm{LD}_{2}$, ortho & 1.0 & $2.7 \cdot 10^{10}$ & 3030 & 1670 & 1.8 \\
\hline $\mathrm{LD}_{2}$, ortho & 1.5 & $2.5 \cdot 10^{10}$ & 3180 & 1600 & 2.0 \\
\hline $\mathrm{LD}_{2}$, para & 1.0 & $1.9 \cdot 10^{10}$ & 2300 & 1150 & 2.0 \\
\hline $\mathrm{LD}_{2}$, para & 1.5 & $1.7 \cdot 10^{10}$ & 2210 & 1070 & 2.1 \\
\hline
\end{tabular}

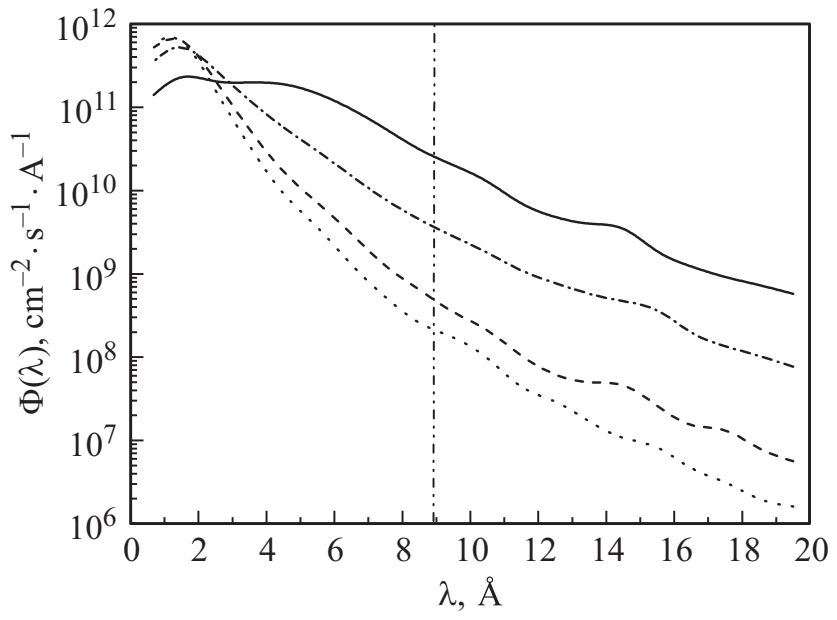

Pис. 4. Спектр нейтронов в гелиевой камере для различных материалов в камере предзамедлителя. Пунктирная кривая спектр в пустой гелиевой камере, в дейтериевой камере газообразный гелий; штриховая кривая - тоже, но в гелиевой камере гелий при температуре $1 \mathrm{~K}$; штрихпунктирная кривая - в гелиевой камере гелий при температуре $1 \mathrm{~K}$, в дейтериевой камере - газообразный водород при температуре $30 \mathrm{~K}$ и давлении $3 \mathrm{~atm}$; сплошная кривая - в дейтериевой камере жидкий ортодейтерий.

Расчетная скорость генерации УХН в гелиевой камере приведена в таблице с учетом и без учета многофононности. Учет многофононности увеличивает скорость генерации УХН в 3.4 раза при температуре гелия $1.0 \mathrm{~K}$ и в 4.1 раза при температуре гелия $1.5 \mathrm{~K}$. Больший эффект от многофононности при температуре гелия $1.5 \mathrm{~K}$ вызван более жестким спектром нейтронов при этой температуре гелия. Ожидаемая скорость генерации УХН в источнике без предзамедлителя составляет порядка $100 \mathrm{~cm}^{-3} \mathrm{~s}^{-1}$ и почти на порядок больше скорости генерации УХН на источнике в Гренобле.

\section{2. Предзамедлитель $\mathrm{H}_{2}, \mathrm{D}_{2}$ или $\mathrm{Be}$}

Различие водорода или дейтерия в качестве рабочих тел состоит в их различной замедляющей способности. Замедляющая способность водорода существенно лучше, чем дейтерия, поэтому для получения сравнимых характеристик источников масса дейтерия в камере предзамедлителя должна быть существенно больше. Однако использование больших масс водорода не эффективно, поскольку водород обладает значительным сечением поглощения нейтронов и при больших размерах камеры с жидким водородом они в основном теряются в ней, поглощаясь водородом. Заполнение дейтериевой камеры газообразным водородом позволяет ограничить массу водорода в камере. Температура водорода должна быть выше температуры кипения. В расчетах температура водорода полагалась равной $30 \mathrm{~K}$, а давление $3 \mathrm{~atm}$. Водород рассматривался в орто- или парасостоянии. Расчет выполнялся для двух температур гелия в камере - 1.0 и $1.5 \mathrm{~K}$. Кроме водорода рассматривалось заполнение камеры предзамедлителя газообразным дейтерием в орто- или парасостоянии также при температуре $30 \mathrm{~K}$. Результаты расчетов приведены в таблице. Максимальная скорость генерации УХН наблюдается для заполнения камеры ортоводородом. Она достигает величины больше $500 \mathrm{UCN} /\left(\mathrm{cm}^{3} \mathrm{~s}\right)$. Фактор усиления за счет многофононности меняется от 2.2 до 2.3 в зависимости от температуры гелия. Для параводорода скорость генерации УХН порядка $400 \mathrm{UCN} /\left(\mathrm{cm}^{3} \mathrm{~s}\right)$. Заполнение же дейтериевой камеры газообразным дейтерием практически не меняет скорость генерации УХН в гелиевой камере (рост около 30\% по сравнению с вакуумом).

Дополнительно был проведен расчет скорости генерации УХН в гелии при условии, что дейтериевая камера заполнена поликристаллическим бериллием при температуре $20 \mathrm{~K}$. Результаты расчетов приведены в таблице. Здесь скорость генерации увеличивается вдвое. 


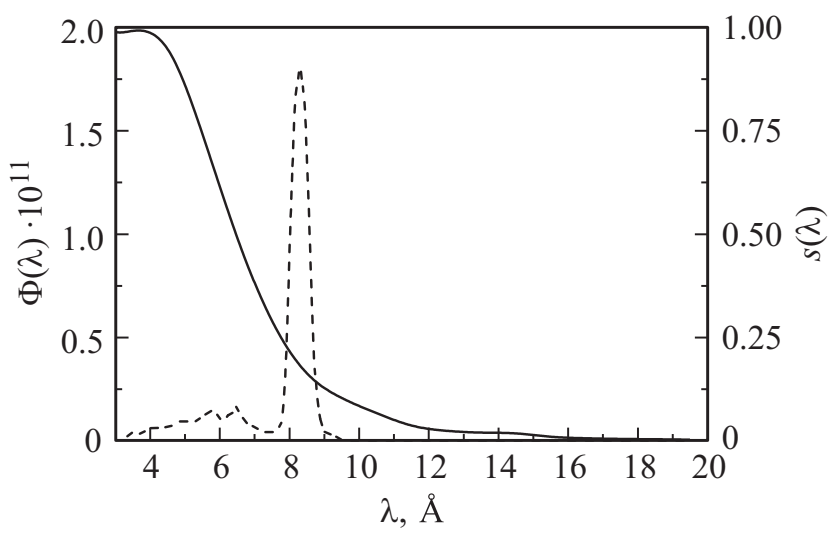

Рис. 5. Зависимость плотности потока нейтронов в гелиевой камере (сплошная кривая, левая шкала) и структурного фактора для гелия от длины волны нейтрона (штриховая линия, правая шкала).

\section{3. Предзамедлитель $\mathrm{LD}_{2}$}

Скорость генерации УХН при заполнении камеры предзамедлителя жидким дейтерием представлена в таблице для случая орто- и парадейтерия для температуры гелия 1.0 и $1.5 \mathrm{~K}$. Максимальную скорость генерации дает жидкий ортодейтерий, при этом она практически не зависит от температуры гелия и достигает величины больше $3000 \mathrm{VXH}$ в USN $/\left(\mathrm{cm}^{3} \mathrm{~s}\right)$. Спектр нейтронов в гелиевой камере в зависимости от длины волны для этого случая сравнивается со структурным фактором на рис. 5. Переход с ортодейтерия на парадейтерий уменьшает скорость генерации в 1.5 раза. Влияние температуры гелия в этом случае также незначительно.

\section{3. Плотность УХН в экспериментальной ловушке для источника с LD $_{2}$ предзамедлителем}

Для определения плотности УХН, доступной для экспериментов, проведено МК моделирование для варианта заполнения камеры предзамедлителя жидким дейтерием в ортосостоянии [5]. Расчетная схема включает в себя камеру источника, нейтроноводную систему и экспериментальную ловушку. На рис. 6 показана расчетная плотность УХН в зависимости от температуры жидкого гелия в камере источника. Верхняя кривая на рис. 6 показывает плотность УХН, которая могла бы получиться в закрытой камере источника в результате накопления предельной плотности УХН. Предельная плотность УХН определяется произведением скорости генерации УХН на время хранения УХН в закрытой камере источника. Однако мы не планируем работать в накопительном режиме с затвором, так как это не дает выигрыша. Соотношение объема всей системы (источник, нейтроновод, экспериментальная ловушка) к

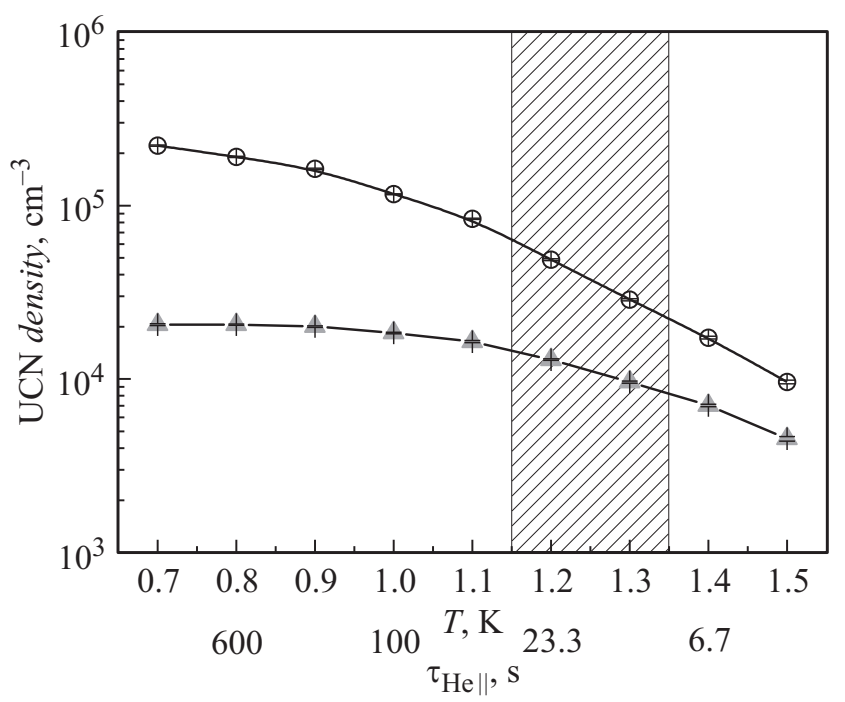

Рис. 6. Плотность УХН в зависимости от температуры жидкого гелия в камере источника для варианта заполнения камеры предзамедлителя жидким дейтерием в ортосостоянии: ○ - в закрытой камере источника, $\boldsymbol{\Delta}$ - в ловушке ЭДМ спектрометра.

объему источника составляет приблизительно 3 раза, соответственно как минимум в 3 раза падает плотность УХН при заполнении экспериментальной ловушки. Стационарный режим при заполнении экспериментальной ловушки проще и надежнее. Нижняя кривая показывают плотность УХН в ловушке эксперимента по измерению электрического дипольного момента (ЭДМ) нейтрона (351) в режиме стационарно работающего источника [6]. Вдоль оси абсцисс дополнительно отложены значения времени хранения нейтронов в жидком гелии при данной температуре [7-9]. Время хранения УХН в жидком гелии падает с ростом его температуры, что приводит к снижению достижимой плотности УХН. Плотность УХН в ловушке эксперимента по измерению ЭДМ нейтрона составит $\sim 1.3 \cdot 10^{4} \mathrm{n} / \mathrm{cm}^{3}$ при температуре жидкого гелия $1.2 \mathrm{~K}$. Это более чем на два порядка превышает плотность УХН на реакторе Института Лауэ-Ланжевена.

\section{Заключение}

В настоящей работе были рассчитаны скорости генерации УХН в источнике на реакторе ВВР-М при различном заполнении камеры предзамедлителя. Различное заполнении соответствует разной степени термализации нейтронов, так что только жидкий дейтерий обеспечивает требуемую степень термализации с максимальным количеством нейтронов с длиной волны $8.9 \AA$ А. Нейтроны с меньшей длиной волны могут генерировать УХН за счет многофононных процессов. Вклад многофононных процессов меньше для более холодного спектра.

Исследовалась зависимость скорости генерации УХН от температуры гелия. При заполнении камеры предза- 
медлителя жидким дейтерием в ортосостоянии скорость генерации УХН практически постоянна в диапазоне изменения температуры гелия от 1.0 до $1.5 \mathrm{~K}$ и достигает $3100 \mathrm{UCN} /\left(\mathrm{cm}^{3} \mathrm{~s}\right)$. При этом плотность УХН в ловушке эксперимента по измерению ЭДМ нейтрона составит $\sim 1.3 \cdot 10^{4} \mathrm{n} / \mathrm{cm}^{3}$ при температуре жидкого гелия $1.2 \mathrm{~K}$. Переход на газообразный водород приводит к уменьшению скорости образования УХН в камере приблизительно в 6 раз.

Исследование выполнено в ФГБУ ПИЯФ НИЦ КИ за счет гранта Российского научного фонда (проект № 1422-00105).

\section{Список литературы}

[1] Serebrov A.P. // Crystallogr. Rep. 2011. Vol. 56. N 7. P. 1230.

[2] Korobkina E. et al. // Phys. Lett. A. 2002. Vol. 301. P. 462.

[3] MacFarlane R.E., Muir D.W. The NJOY Nuclear Data Processing System. Препринт Лос-Аламосской лаборатории CШA: LA-12740-M. 1994.

[4] Farhi E. Institute Laue-Langevin. (Частное сообщение)

[5] Серебров А.П., Фомин А.К. // ЖТФ. 2015. Т. 85. Вып. 8. C. 136.

[6] Serebrov A.P. et al. // Phys. Rev. C. 2015. Vol. 92. P. 055501.

[7] Golub R. et al. // Z. Phys. B. Condensed Matter. 1983. Vol. 51. P. 187.

[8] Baker C.A. et al. // Phys. Lett. A. 2003. Vol. 308. P. 67.

[9] Leung K.K.H. et al. // Phys. Rev. C. 2016. Vol. 93. P. 025501. 